\title{
Ubiquitin-specific protease 53 promotes osteogenic differentiation of human bone marrow-derived mesenchymal stem cells
}

\author{
Dawoon Baek ${ }^{1,2}$, Kwang Hwan Park', Kyoung-Mi Lee ${ }^{1,3}$, Sujin Jung ${ }^{1,2}$, Soyeong Joung ${ }^{1,2}$, Jihyun Kim ${ }^{1,2}$ and
} Jin Woo Lee (1) ${ }^{1,2,3}$

\begin{abstract}
The ubiquitin protease pathway plays important role in human bone marrow-derived mesenchymal stem cell (hBMSC) differentiation, including osteogenesis. However, the function of deubiquitinating enzymes in osteogenic differentiation of hBMSCs remains poorly understood. In this study, we aimed to investigate the role of ubiquitinspecific protease 53 (USP53) in the osteogenic differentiation of hBMSCs. Based on re-analysis of the Gene Expression Omnibus database, USP53 was selected as a positive regulator of osteogenic differentiation in hBMSCs. Overexpression of USP53 by lentivirus enhanced osteogenesis in hBMSCs, whereas knockdown of USP53 by lentivirus inhibited osteogenesis in hBMSCs. In addition, USP53 overexpression increased the level of active $\beta$-catenin and enhanced the osteogenic differentiation of hBMSCs. This effect was reversed by the Wnt/ $\beta$-catenin inhibitor DKK1. Mass spectrometry showed that USP53 interacted with F-box only protein 31 (FBXO31) to promote proteasomal degradation of $\beta$-catenin. Inhibition of the osteogenic differentiation of hBMSCs by FBXO31 was partially rescued by USP53 overexpression. Animal studies showed that hBMSCs with USP53 overexpression significantly promoted bone regeneration in mice with calvarial defects. These results suggested that USP53 may be a target for gene therapy for bone regeneration.
\end{abstract}

\section{Introduction}

Bone homeostasis is regulated by the balance of osteogenesis (bone formation) and osteoclastogenesis (bone resorption $)^{1-3}$. The imbalance of these processes leads to low bone density and deterioration of the bone matrix, which increases the risk of skeletal disorders, such as osteoporosis $^{4}$. Osteoporosis causes serious socioeconomic problems $^{5,6}$. Several therapeutics have been developed to maintain bone homeostasis ${ }^{7,8}$, including bone-forming and antiresorptive compounds. However, these compounds have serious side effects, including peptic ulcers,

\footnotetext{
Correspondence: Jin Woo Lee (ljwos@yuhs.ac)

'Department of Orthopaedic Surgery, Yonsei University College of Medicine, 50-1 Yonsei-ro, Seodaemun-gu, Seoul 03722, South Korea

${ }^{2}$ Brain Korea 21 PLUS Project for Medical Sciences, Yonsei University College of Medicine, 50-1 Yonsei-ro, Seodaemun-gu, Seoul 03722, South Korea

Full list of author information is available at the end of the article These authors contributed equally: Dawoon Baek, Kwang Hwan Park Edited by Y. Wang
}

atypical femur fractures, osteonecrosis of the jaws, and increased risk of bone tumor ${ }^{9,10}$. Therefore, novel therapeutic agents are urgently needed.

The ubiquitin-proteasome pathway (UPP) is an ATPdependent, reversible pathway that degrades target proteins ${ }^{11}$. Up to $80 \%$ of all intracellular proteins are degraded through the UPP. The reaction occurs through the hierarchal transfer of ubiquitin-activating enzyme (E1), ubiquitin-conjugating enzyme (E2), and ubiquitin ligase (E3), resulting in target protein degradation ${ }^{11}$. Deubiquitinating enzymes (DUBs) then remove ubiquitin chains from a target protein, controlling the function and stability of the protein ${ }^{12,13}$.

UPPs have important roles in the osteogenic differentiation of human bone marrow-derived mesenchymal stem cells (hBMSCs) ${ }^{14}$. Runt-related transcription factor 2 (RUNX2), a master transcription factor in osteogenesis, interacts with smad ubiquitination regulatory factor 1

\section{(c) The Author(s) 2021}

\footnotetext{
(c) Open Access This article is licensed under a Creative Commons Attribution 4.0 International License, which permits use, sharing, adaptation, distribution and reproduction cc in any medium or format, as long as you give appropriate credit to the original author(s) and the source, provide a link to the Creative Commons license, and indicate if changes were made. The images or other third party material in this article are included in the article's Creative Commons license, unless indicated otherwise in a credit line to the material. If material is not included in the article's Creative Commons license and your intended use is not permitted by statutory regulation or exceeds the permitted use, you will need to obtain permission directly from the copyright holder. To view a copy of this license, visit http://creativecommons.org/licenses/by/4.0/.
} 
a

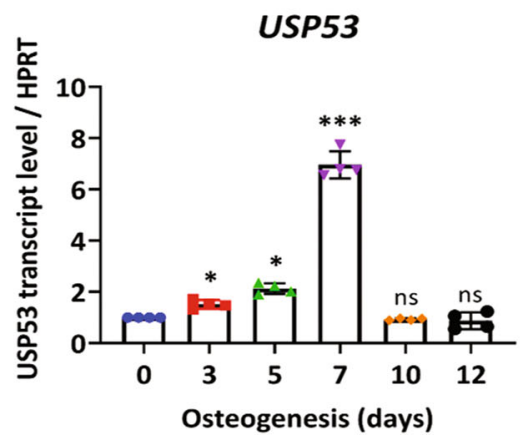

b

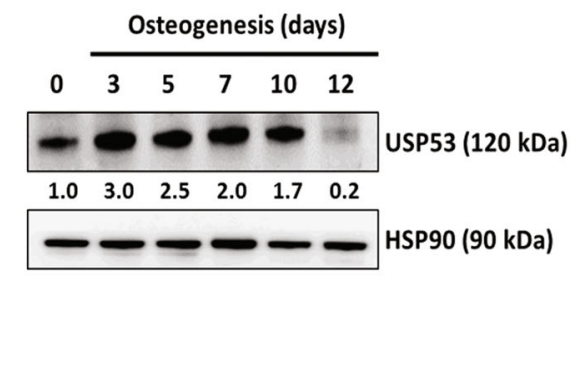

Fig. 1 Upregulation of USP53 during the osteogenic differentiation of hBMSCs. a Relative mRNA expression levels of USP53 during the osteogenic differentiation of hBMSCs. HPRT was used for normalization. b Protein levels of USP53 during the osteogenic differentiation of hBMSCs. Heat shock protein 90 (HSP90) was used as a loading control. USP53 protein expression was quantified using ImageJ. Day 0: undifferentiated hBMSCs. Results are means \pm SDs. ns: not significant, ${ }^{*} P<0.05,{ }^{* *} P<0.001$ by one-way ANOVA. $n=3$.

(Smurf1) and Smurf2, E3 ligases, leading to the degradation of RUNX2 ${ }^{15}$. As an E3 ligase, WW domaincontaining E3 ubiquitin protein ligase 1 negatively regulates osteoblast differentiation targeting RUNX2 ${ }^{16}$. The E3 ligase, Itch, also negatively regulates osteoblast function ${ }^{17}$. Accumulating evidence indicates that E3 ligases play important roles in the regulation of osteogenesis in hBMSCs $^{18,19}$. However, few studies have addressed the functions of DUBs in the osteogenic differentiation of hBMSCs $^{20,21}$.

Ubiquitin-specific peptidase 53 (USP53), a member of the deubiquitinating enzyme family, contains a catalytically inactive ubiquitin-specific protease domain and is expressed in cochlear hair cells ${ }^{22}$. Mice lacking Usp53 exhibit progressive hearing $\operatorname{loss}^{22}$; additionally, USP53 is mutated in novel syndromic forms of cholestatic liver disease in humans ${ }^{23}$. However, the functions of USP53 are unclear.

Accordingly, in this study, we identified USP53 as a differentially expressed gene (DEG) in re-analysis of the Gene Expression Omnibus (GEO) database and investigated the roles of USP53 in osteogenic differentiation of hBMSCs. We hypothesized that USP53 may positively regulate the osteogenic differentiation of hBMSCs in vitro and that local delivery of AAV2-USP53 to calvarial defects would thus enhance bone formation. Our findings provide important insights into the potential applications of AAV2-USP53-activated hBMSCs in gene therapy for bone regeneration.

\section{Results}

Expression of upregulated DUBs during the osteogenic differentiation of hBMSCs and selection of USP53 as a DUB-associated molecule

Based on GEO datasets and previous studies ${ }^{24}$, commonly upregulated DUBs in hBMSC osteogenesis were analyzed (Supplementary Table S1). From the selected DUBs, USP53 was found to show the most marked upregulation during the osteogenic differentiation of hBMSCs. Next, we examined the relative mRNA and protein levels of osteogenic markers during osteogenesis in hBMSCs. The mRNA and protein expression levels of osteogenic markers increased in the early phase of hBMSC osteogenesis (days 3-7) and decreased during the late phase of hBMSC osteogenesis (days 10-12; Supplementary Fig. 1). Consistent with the database findings, USP53 mRNA and protein expression levels were increased in the early phase and decreased in the late phase of osteogenesis in hBMSCs (Fig. 1a, b). Thus, USP53 may regulate the osteogenic differentiation of hBMSCs.

\section{Knockdown of USP53 inhibited the osteogenic differentiation of hBMSCs in vitro}

We then investigated the roles of USP53 in the osteogenic differentiation of hBMSCs using short hairpin RNA (shRNA) targeting USP53. Approximately 90\% knockdown efficiency was achieved (Supplementary Fig. 2a, b). Additionally, knockdown of USP53 decreased ALP and Alizarin Red S staining intensity as well as ALP activity (Fig. 2a-d). The effects of USP53 knockdown on osteogenic differentiation were not attributable to cell viability (Supplementary Fig. 3a, b). qRT-PCR and western blotting revealed that osteogenic marker levels in USP53knockdown hBMSCs were decreased compared with that in shMock-transfected cells (Fig. 2e, f). These results suggested that knockdown of USP53 decreased the osteogenic differentiation of hBMSCs.

\section{Overexpression of USP53 promoted the osteogenic differentiation of hBMSCs in vitro}

To further examine the roles of USP53 in the osteogenic differentiation of hBMSCs, we prepared lentiviral 
a

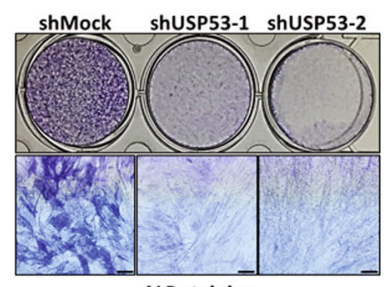

ALP staining

(hBMSC, Osteogenesis day 3)

c

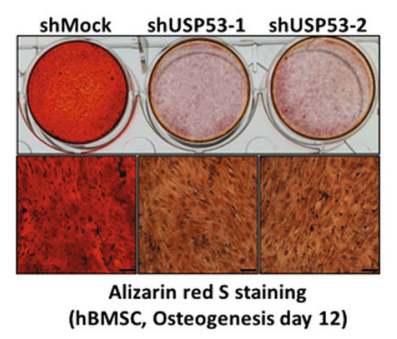

b

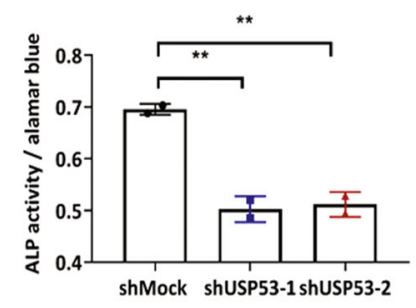

d

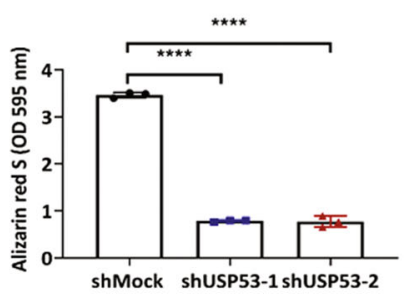

e

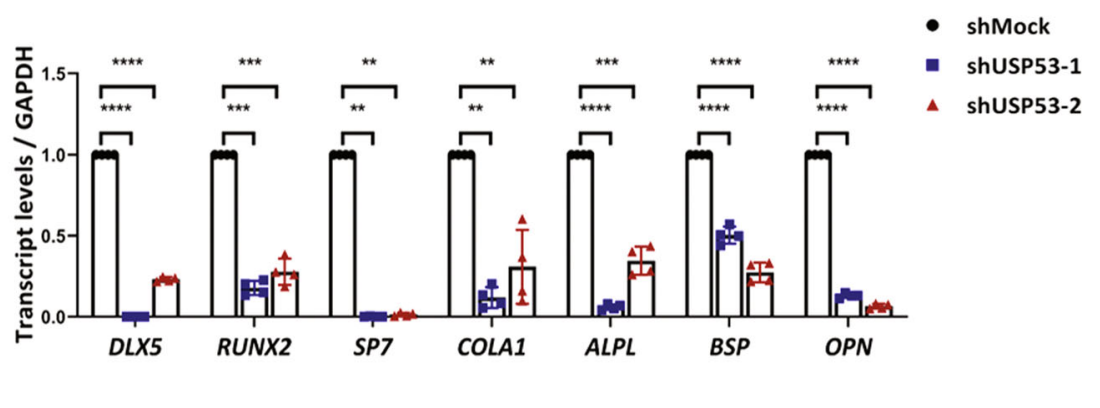

$\mathbf{f}$

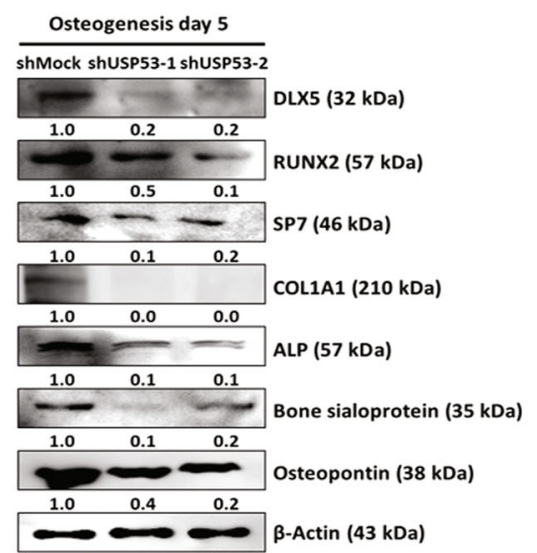

Fig. 2 USP53 knockdown inhibits the osteogenic differentiation of hBMSCs in vitro. a Alkaline phosphatase staining was performed in shMockor shUSP53-1-, 2-infected hBMSCs in osteogenic medium for 3 days. Scale bars, $200 \mu \mathrm{m}$. b Quantification of ALP activity. c Alizarin red S staining was performed in shMock- or shUSP53-1-, 2-infected hBMSCs in osteogenic medium for 12 days. Scale bars, $200 \mu$ m. d Quantification of Alizarin red S staining. e qRT-PCR analysis of osteogenesis-related genes on day 3 after osteogenic differentiation. $\mathbf{f}$ Immunoblot analysis of osteogenesis-related genes on day 5 after osteogenic differentiation. Data were quantified using ImageJ. Results are means \pm SDs. ns: not significant, ${ }^{* *} P<0.01,{ }^{* * *} P<$ $0.001,{ }^{* * *} P<0.0001$ by one-way ANOVA. $n=3$.

constructs expressing USP53 in hBMSCs. High overexpression efficiency was confirmed in USP53overexpressing hBMSCs (Supplementary Fig. 2c, d). Overexpression of USP53 enhanced ALP and Alizarin red $S$ staining intensity as well as ALP activity (Fig. 3a-d). The effects of overexpression of USP53 on osteogenic differentiation were not attributable to cell viability (Supplementary Fig. 3c, d). qRT-PCR and 
a

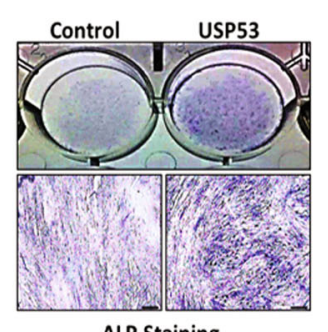

ALP Staining

(hBMSC, Osteogenesis day 3)

c

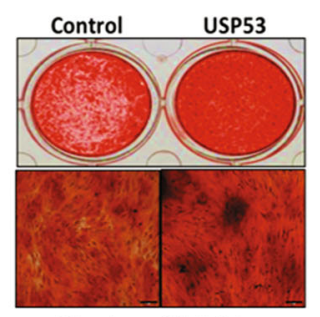

Alizarin red $\mathrm{S}$ staining (hBMSC, Osteogenesis day 12) b

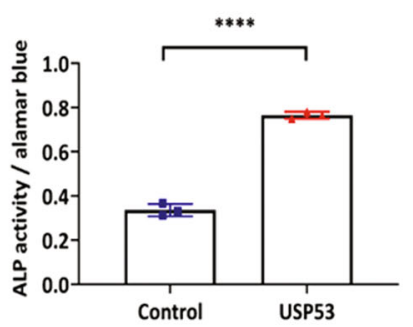

d

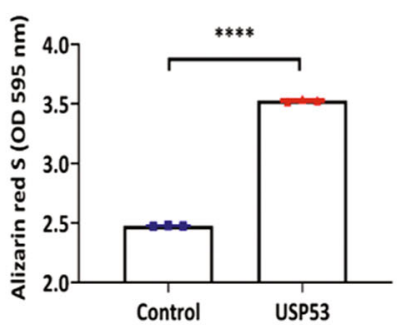

e
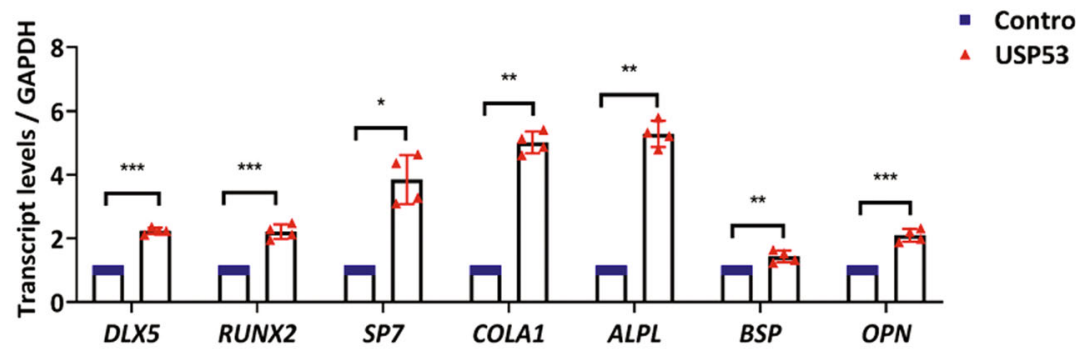

f

Osteogenesis day 5

Control USP53

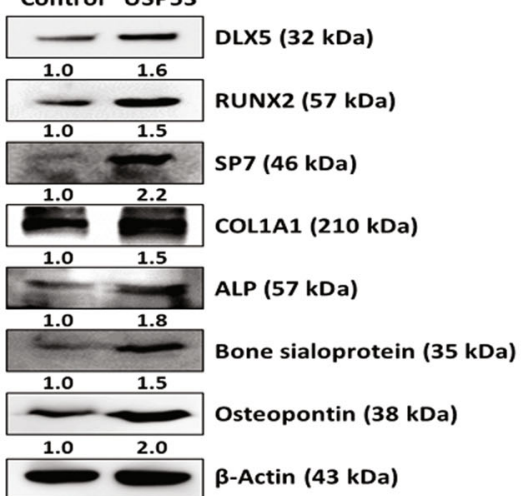

Fig. 3 USP53 overexpression promotes the osteogenic differentiation of hBMSCs in vitro. a Alkaline phosphatase staining was performed in control or USP53-infected hBMSCs in osteogenic medium for 3 days. Scale bars, $200 \mu \mathrm{m}$. b Quantification of ALP activity. c Alizarin red S staining was performed in osteogenic medium for 12 days. Scale bars, $200 \mu \mathrm{m}$. d Quantification of Alizarin red S staining. e qRT-PCR analysis of osteogenesis-related genes on day 3 after osteogenic differentiation. $\mathbf{f}$ Immunoblot analysis of osteogenesis-related genes on day 5 after osteogenic differentiation. Data were quantified using ImageJ. Results are means \pm SDs. ${ }^{*} P<0.05,{ }^{* *} P<0.01,{ }^{* *} P<0.001,{ }^{* * *} P<0.0001$ by unpaired two-tailed Student's $t$-test. $n=3$. 
western blot analysis revealed that osteogenic marker expression levels in USP53-overexpressing hBMSCs were upregulated compared with those in control cells (Fig. 3e, f). Thus, USP53 positively regulated the osteogenic differentiation of hBMSCs.

\section{USP53 regulated the osteogenic differentiation of hBMSCs through Wnt/ß-catenin signaling}

A signaling pathway involving $\mathrm{Wnt} / \beta$-catenin, transforming growth factor-beta (TGF- $\beta$ ), bone morphogenetic protein (BMP), parathyroid hormone-related protein (PTHrP), and insulin-like growth factor (IGF) signaling plays important roles in regulating RUNX2 expression. Thus, western blot analysis was performed to evaluate the expression of $\mathrm{Wnt} / \beta$-catenin signal pathway mediators in hBMSCs with overexpression or knockdown of USP53. The levels of unphosphorylated $\beta$-catenin were higher in hBMSCs overexpressing USP53 than in the control (Fig. 4a). Conversely, the levels of unphosphorylated $\beta$-catenin in USP53-knockdown hBMSCs were reduced compared with those in shMock-transfected cells (Fig. 4b). There were no significant differences in other signaling pathways (Supplementary Figs. 4 and 5 and Table S2).

To investigate whether USP53 promoted the osteogenesis of hBMSCs through the Wnt/ $\beta$-catenin signaling pathway, we examined the effects of a Wnt/ $\beta$-catenin activator (Wnt3a) and inhibitor (DKK1) in hBMSCs with knockdown or overexpression of USP53. Inhibition of the osteogenic differentiation capacity in the shUSP53 group was rescued in response to Wnt3a, an activator of the Wnt pathway (Fig. 4c). Conversely, enhanced osteogenic differentiation capacity in the USP53 overexpression group was reduced in response to DKK1, an inhibitor of the Wnt pathway (Fig. 4d), suggesting that USP53 may be essential for the osteogenic differentiation of hBMSCs through $\mathrm{Wnt} / \beta$-catenin signaling. In vitro ubiquitination assays showed that $\beta$-catenin degradation was significantly reduced by USP53 overexpression in a concentration-dependent manner, whereas downregulation of USP53 promoted the degradation of $\beta$-catenin (Fig. 4e, f). Moreover, USP53 increased the half-life of active $\beta$-catenin compared with that in the control group (Fig. 4g). Taken together, these findings suggested that USP53 acted as a deubiquitinase enzyme, stabilizing $\beta$-catenin.

Identification of USP53 binding proteins and regulation of $\beta$-catenin degradation by FBXO31 through the Skp1/Cul1/ F-box protein (SCF) complex

Immunoprecipitation (IP) and LC-MS/MS were performed to identify proteins that interacted with USP53 in the osteogenesis of hBMSCs (Supplementary Table S3). Seven proteins were identified as interacting with USP53 during hBMSC osteogenesis, including F-box only protein 31 (FBXO31) (Fig. 5a). To examine whether USP53 interacted with FBXO31 in hBMSCs, co-IP and western blotting were performed using wild-type and mutant FBXO31 (myc-FBXO31 $\Delta$ F), the latter of which harbored a deletion of the F-box domain, which mediates binding to Skp1 and Cul1. As shown in Fig. 5b, USP53 and FBXO31 bound with each other in hBMSCs. However, the FBXO31 mutant failed to interact with USP53, identifying FBXO31 as a novel binding partner of USP53.

FBXO31 is a candidate tumor-suppressor gene in breast, ovarian, hepatocellular, and prostate cancers ${ }^{25}$ and acts as a functional SCF-FBXO31 E3 ubiquitin ligase ${ }^{25,26}$. Therefore, we examined the effects of FBXO31 on the osteogenic differentiation of hBMSCs by controlling the expression of genes associated with osteogenesis. First, we examined the mechanism through which FBXO31 regulated the stability of $\beta$-catenin, a key mediator of the Wnt signaling pathway ${ }^{27}$ and bone formation during osteogenic differentiation ${ }^{28}$, using co-IP and western blot assays with wild-type FBXO31 and myc-FBXO31 $\triangle \mathrm{F}$. As shown in Fig. 5b, FBXO31 specifically interacted with $\beta$-catenin, Skp1, and USP53. These results were effectively reversed by FBXO31 mutation (myc-FBXO31 $\Delta \mathrm{F})$. Ectopic expression of FBXO31 or FBXO31 mutant at increasing concentrations in hBMSCs showed that FBXO31 significantly decreased $\beta$-catenin levels in a concentration-dependent manner; no changes were observed for the FBXO31 mutant (Fig. 5c, d). Moreover, FBXO31-mediated degradation of $\beta$-catenin was significantly blocked in the presence of MG132 (a 26S proteasome inhibitor), indicating that $\beta$-catenin stability was regulated at the posttranslational level by FBXO31 (Fig. 5e).

F-box proteins bind with Skp1 to form an SCF complex through the F-box domain and bring their substrates to the complex for ubiquitination ${ }^{29}$. Therefore, to determine whether FBXO31 degraded $\beta$-catenin through the SCF complex, wild-type and mutant FBXO31 were overexpressed in hBMSCs. As shown in Fig. 5f, compared with wild-type FBXO31, FBXO31 mutant did not reduce $\beta$-catenin levels, indicating that FBXO31 degraded $\beta$-catenin through the SCF complex. Then, we examined whether FBXO31 regulated the transcriptional activity of $\beta$-catenin during the osteogenesis of hBMSCs using TOP/ FOP assays. FBXO31 reduced the transcriptional activity of $\beta$-catenin during the osteogenesis of hBMSCs; however, no significant reduction was observed in the FBXO31 mutant during the same process (Fig. 5g). Finally, $\beta$-catenin polyubiquitination was confirmed by ubiquitination assays. FBXO31 mutant caused FBXO31 to lose the capability to promote $\beta$-catenin degradation (Fig. $5 \mathrm{~h}$ ). These results demonstrated that FBXO31 interacted with $\beta$-catenin and promoted the degradation of $\beta$-catenin through the SCF complex. 
a

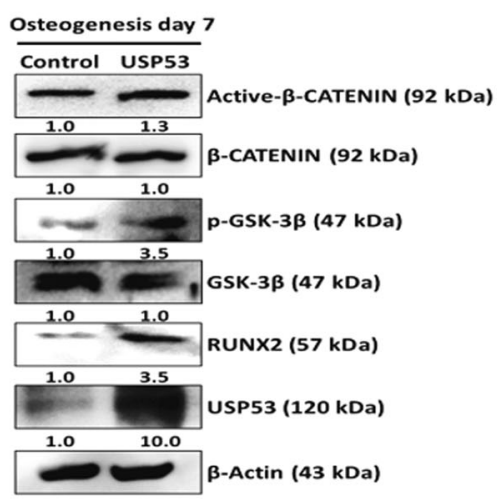

c

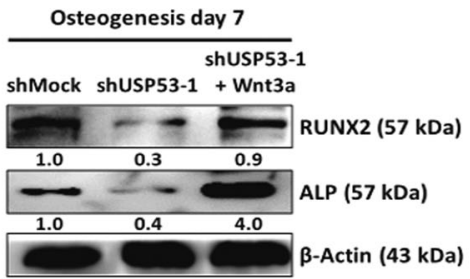

e

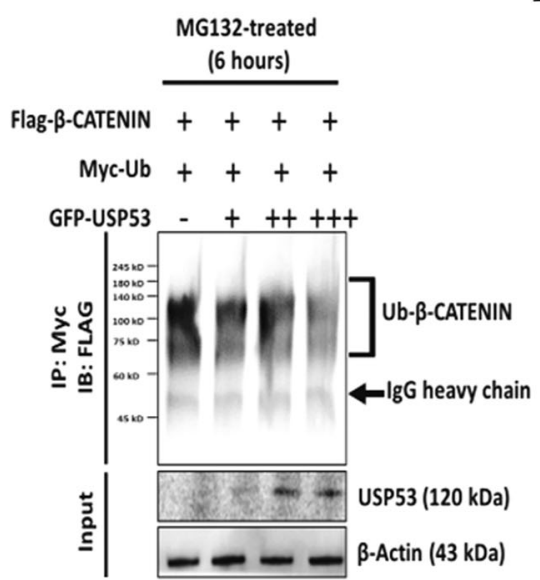

b

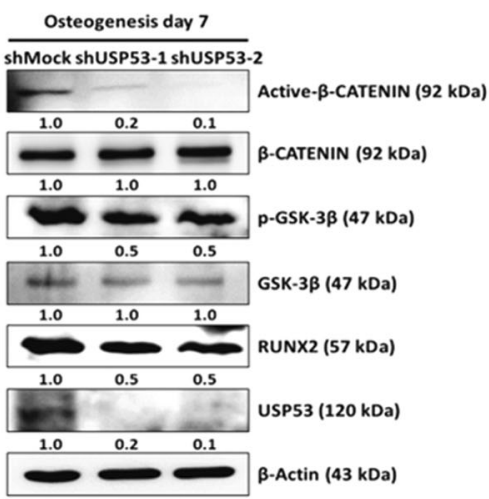

d

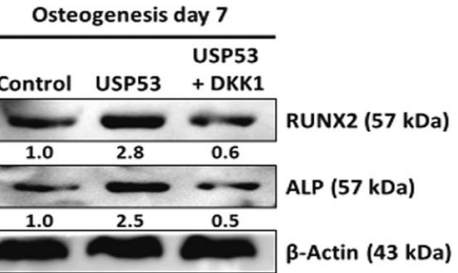

f

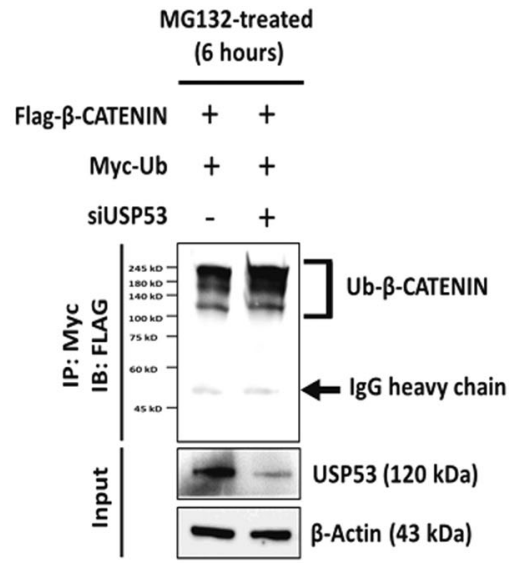

g

+ Wnt3a

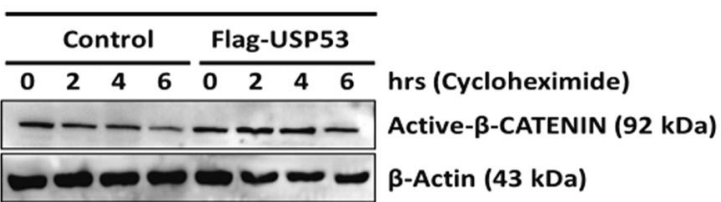

Fig. 4 USP53 regulates osteogenic differentiation in hBMSCs through $\mathbf{W n t} / \boldsymbol{\beta}$-catenin signaling. $\mathbf{a}$, $\mathbf{b}$ Expression levels of Wnt/ $\beta$-catenin signaling pathway mediators were investigated by immunoblotting in USP53-overexpressing (a) or -knockdown (b) hBMSCs in osteogenic medium for 7 days. Data were quantified using ImageJ. c Immunoblot analysis of Wnt3a-induced osteogenic marker expression. Data were quantified using ImageJ. d Immunoblot analysis of DKK1-induced osteogenic marker expression. Data were quantified using ImageJ. e Immunoblot analysis of $\beta$-catenin-linked polyubiquitination with overexpression of USP53. $\beta$-Catenin ubiquitination (top panel) and protein expression assays (bottom panel) were evaluated. $\mathbf{f}$ Immunoblot analysis of $\beta$-catenin-linked polyubiquitination with siUSP53. $\beta$-Catenin ubiquitination (top panel) and protein expression assays (bottom panel) were evaluated. $\mathbf{g}$ hBMSCs were transfected with Flag- $\beta$-catenin overexpression plasmid and control or Flag-USP53 and then treated with cycloheximide $(100 \mu \mathrm{g} / \mathrm{mL})$ and Wnt3a. Immunoblots with active $\beta$-catenin protein at the indicated time points. $n=3$. 


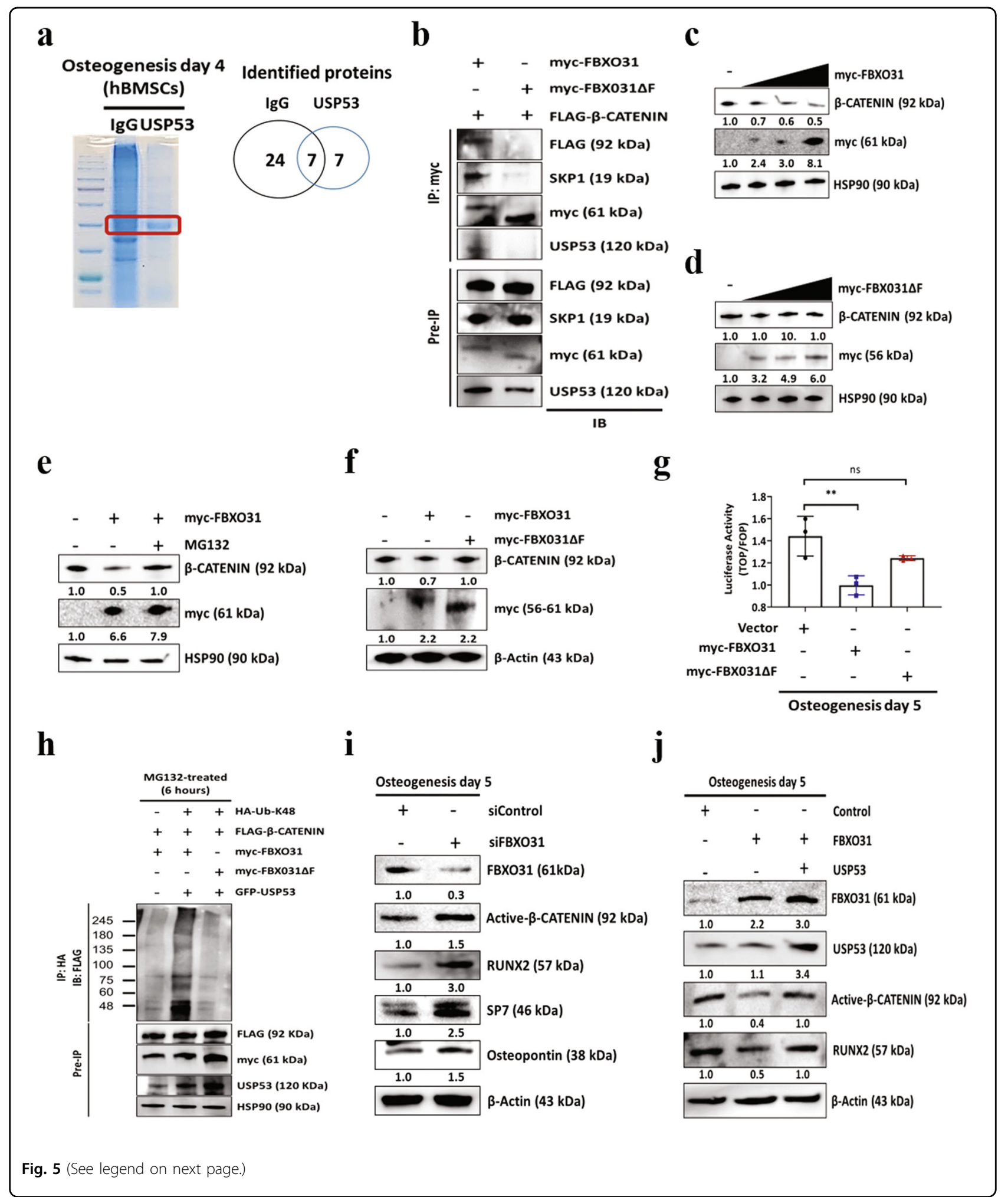

Next, we further investigated the effects of FBXO31 on the osteogenic differentiation of hBMSCs. First, we examined the effects of FBXO31 RNA interference on the osteogenic differentiation potential of hBMSCs. As shown in Fig. 5i, RNA interference of FBXO31 significantly increased active $\beta$-catenin and osteogenic marker levels compared with scrambled control siRNA. Conversely, FBXO31 overexpression decreased active $\beta$-catenin levels, 
(see figure on previous page)

Fig. 5 Identification of USP53 binding proteins and effects of FBXO31 on $\beta$-catenin degradation through the SCF complex. a Instantblue staining of a co-IP mixture separated by SDS-PAGE. The indicated band was extracted for analysis. $\mathbf{b}$ hBMSCs were cotransfected with FLAG- $\beta$-catenin with either myc-FBXO31 or myc-FBXO31 $\triangle \mathrm{F}$ for $48 \mathrm{~h}$. Transfected cells were incubated with $10 \mu \mathrm{M} \mathrm{MG132}$ for $6 \mathrm{~h}$, and whole cells were lysed and subjected to IP with anti-myc antibodies. Immunoprecipitates and input protein extracts (Pre-IP) were resolved in SDS-PAGE. c hBMSCs were transfected with pCMV-myc or myc-FBXO31 for $48 \mathrm{~h}$, and whole cell lysates were immunoblotted. $\mathbf{d}$ hBMSCs were transfected with pCMV-myc or myc-FBXO31 $\triangle \mathrm{F}$ for $48 \mathrm{~h}$, and whole-cell lysates were immunoblotted. e hBMSCs were transfected with the pCMV-myc or myc-FBXO31 for $48 \mathrm{~h}$. Transfected cells were then incubated with or without $10 \mu \mathrm{M}$ MG132 for $6 \mathrm{~h}$. Cell lysates were immunoblotted. f hBMSCs were transfected with pCMV-myc, myc-FBXO31, and myc-FBXO31 $\triangle \mathrm{F}$ for $48 \mathrm{~h}$. Cells were harvested and lysed, and whole-cell protein extracts were immunoblotted. g $\beta$-Catenin transcriptional activity was measured on day 5 after induction of osteogenesis by TOP/FOP luciferase assays. $\mathbf{h}$ HEK293 cells were transfected with the indicated plasmids for $48 \mathrm{~h}$, treated with $10 \mu \mathrm{M} \mathrm{MG132}$ for $6 \mathrm{~h}$, lysed, subjected to IP with anti-HA antibodies, and immunoblotted. i hBMSCs were transfected with negative control or FBXO31 siRNA for 5 days in osteogenic induction medium. Immunoblot analysis was performed, and data were quantified using ImageJ. $\mathbf{j}$ hBMSCs were transfected with empty vector, FBXO31, or GFP-USP53 and then cultured with osteogenic induction medium for 5 days. Immunoblot analysis was performed, and data were quantified using ImageJ. Results are means \pm SDs. ns: not significant, ${ }^{* *} P<0.01$ by one-way ANOVA. $n=3$.

resulting in reduced osteogenesis of hBMSCs. However, this phenomenon was rescued after modulating the expression of USP53 (Fig. 5j). These results demonstrated that the FBXO31-mediated reduction of osteogenesis in hBMSCs was significantly blocked in the presence of USP53.

\section{hBMSCs with AAV2-USP53 improved bone formation in vivo}

A mouse calvarial defect model was used to determine the effects of AAV2-USP53-infected hBMSCs on bone formation in vivo. Critical-size calvarial defects $(5 \mathrm{~mm}$ in diameter) were created and then covered with fibrin glue mixed with AAV2-control-infected hBMSCs or AAV2USP53-infected hBMSCs (Fig. 6a). Eight weeks after surgery, $\mu C T$ images showed enhanced bone regeneration in the AAV2-USP53-infected hBMSC group compared with that in AAV2-control-infected hBMSCs (Fig. 6b). Quantification of $\mu \mathrm{CT}$ images showed that the proportion of bone volume per tissue volume (BV/TV) and bone surface density in the AAV2-USP53-infected hBMSC group were higher than those in the AAV2-control-infected hBMSC group (Fig. 6c). Calvarial surface calcein staining and mineral apposition rates were increased in the AAV2USP53-infected hBMSC group (Fig. 6d).

Next, to investigate new bone formation, we performed M\&T staining. The results showed that newly formed bone in the AAV2-USP53-infected hBMSC group was increased compared with that in the AAV2control-infected hBMSC group (Fig. 6e). Evaluation of the expression of vimentin, a mesenchymal stem cell marker, by IHC showed that vimentin expression was increased in the AAV2-USP53-infected hBMSC group, indicating that the newly regenerated bone originated from the implanted hBMSCs (Fig. 6f). Next, we investigated the expression of osteocalcin $(\mathrm{OCN})$, an important marker of extracellular matrix mineralization, by IHC analysis. The expression of OCN was higher in the AAV2-USP53-infected hBMSC group than in the
AAV2-control-infected hBMSC group (Fig. 6g). Taken together, these results demonstrated that AAV2-USP53infected hBMSCs had therapeutic effects on a calvarial defect murine model.

\section{USP53 expression was decreased in patients and mice with osteoporosis}

Osteoporosis is a disease that causes bones to become weak without symptoms and increases the risk of broken bones. To explore the relationships between USP53 and osteoporosis, a GEO dataset (GSE35959) ${ }^{30}$ was analyzed. The results showed that USP53 expression in BMSCs from patients with osteoporosis was downregulated compared with that in normal controls (Supplementary Table S4). Furthermore, the roles of USP53 in osteoporosis were examined in an OVX mouse model to induce estrogen deficiency-related osteoporosis. $\mu \mathrm{CT}$ results showed that there was a significant reduction in trabecular bone mass in OVX mice compared with that in sham control mice (Fig. 7a). Next, we confirmed the expression of CTX-1 in OVX mice by ELISA. The expression of the bone resorption marker CTX-1 in OVX mice was increased compared with that in sham control mice (Fig. 7b). Moreover, the protein levels of USP53 in bone sections from OVX mice were also lower than those from sham mice, as confirmed by immunofluorescence staining (Fig. 7c). Taken together, these results demonstrated that USP53 expression was downregulated in patients and mice with osteoporosis.

\section{Discussion}

Understanding the molecular mechanisms that regulate bone homeostasis is essential for the development of improved therapeutics to treat human skeletal disorders. UPPs are required for the osteogenic differentiation of hBMSCs $^{14}$. Since USPs regulate cell cycle activity that is involved in post-translational modification, it is crucial in various diseases. Recent studies reported that USPs play an important role in cancer progression. In case of USP2a, 

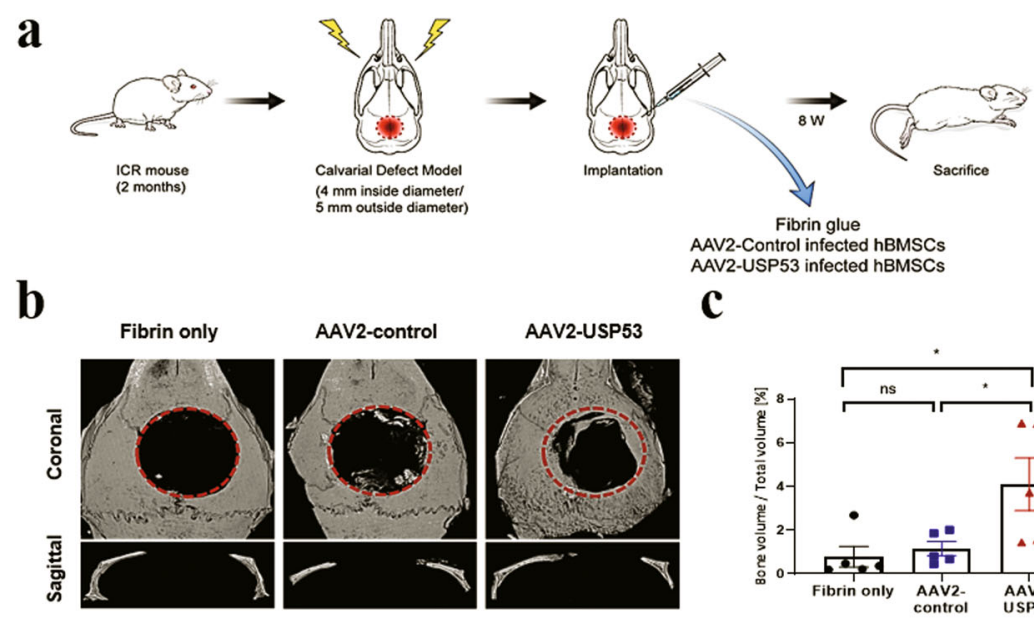

AAV2-USP53 infected hBMSCs
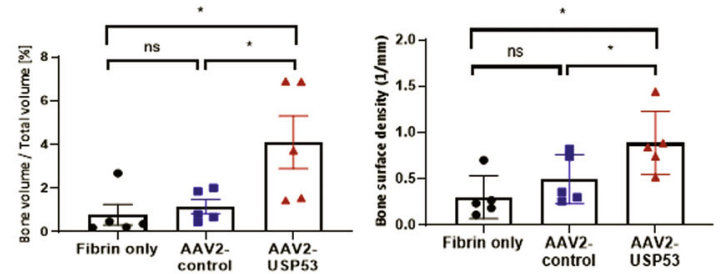

d

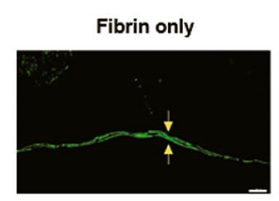

AAV2-control

AAV2-USP53
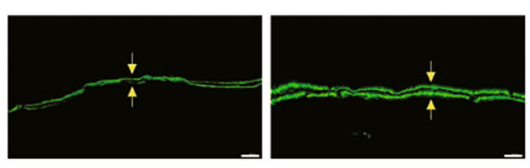

e

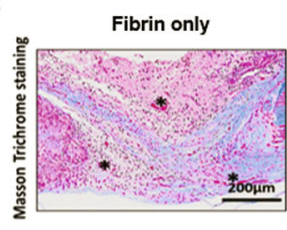

AAV2-control

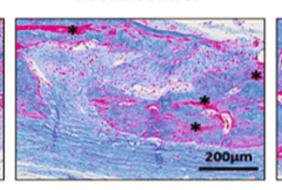

AAV2-USP53

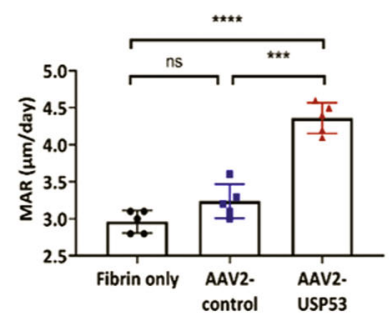

f

g
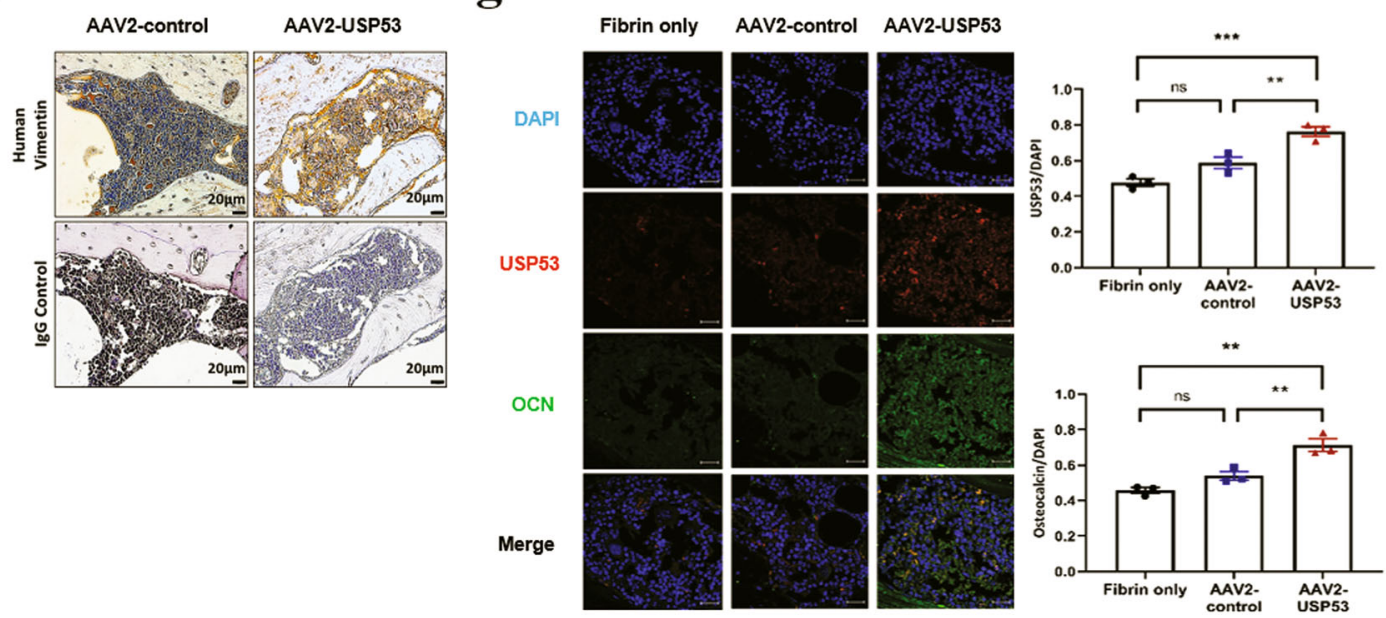

Fig. 6 hBMSCs with AAV2-USP53 improved bone formation in vivo. a Experimental design of the mouse calvarial defect model. b Critical-size calvarial defects (5 mm in diameter) in mice were treated with AAV2-control-infected hBMSCs or AAV2-USP53-infected hBMSCs in fibrin matrix with PBS. Eight weeks after surgery, bone regeneration was measured by $\mu C T$. c Relative quantification of $\mu C T$ analysis. $\mathbf{d}$ Histomorphometric analysis of calvarial defects in mice. Arrows indicate the distance between double calcein labeling. Scale bars, $20 \mu \mathrm{m}$. Relative histomorphometric quantification of the mineral apposition rate (MAR) is shown (right). e Representative images of M\&T staining of calvarial bone sections in mice. Scale bars, $200 \mu \mathrm{m}$. *New bone. $\mathbf{f}$ Immunohistochemistry analysis using an antibody against human vimentin and IgG control of calvarial bone sections in mice. Scale bars, $20 \mu \mathrm{m}$. g Immunohistochemistry analysis of USP53 (phycoerythrin [PE]; red fluorescence) and OCN (fluorescein isothiocyanate [FITC]; green fluorescence) with DAPI counterstaining of the calvarial defects in mice (left). Quantification of IHC analysis (right). Scale bars, $50 \mu \mathrm{m}$. Results are presented as means \pm SDs. ns: not significant, ${ }^{*} P<0.05,{ }^{* *} P<0.01,{ }^{* * *} P<0.001,{ }^{* * *} P<0.0001$ by one-way ANOVA. $n=5$ per group. 
a
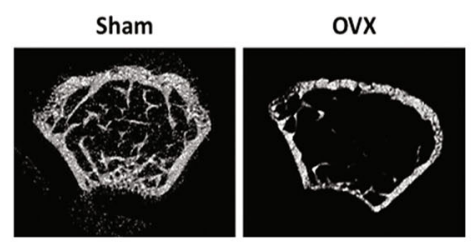

b

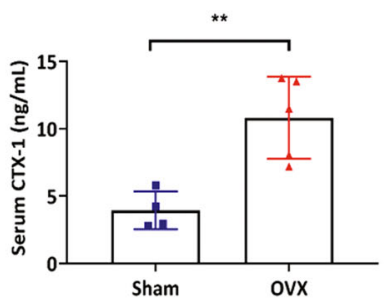

c
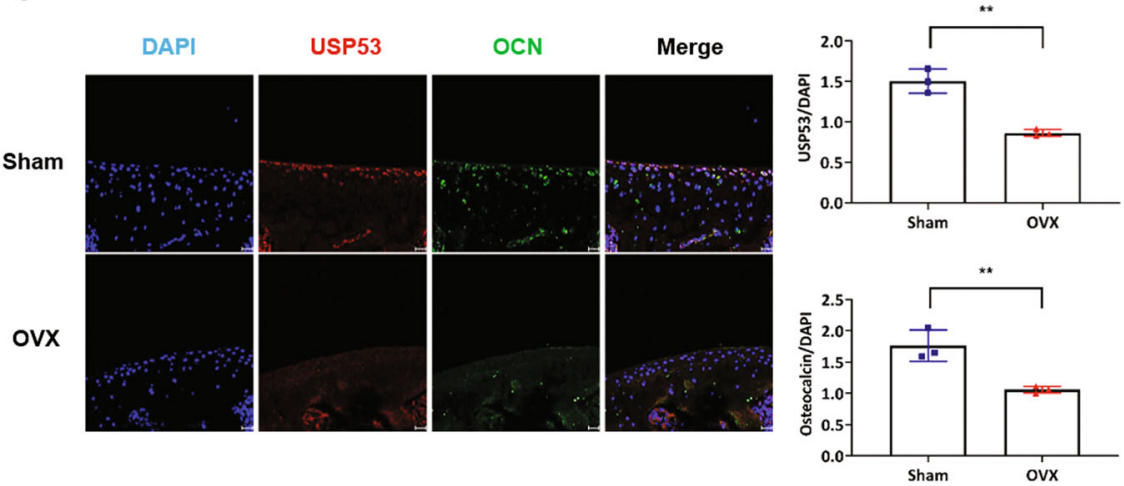

d

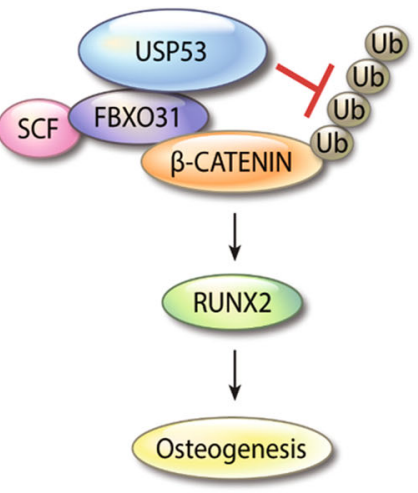

Fig. 7 USP53 levels decrease in an osteoporosis mouse model. a Sham or ovariectomy (OVX) surgery was performed on 6-week-old female mice. Two months later, femoral trabecular bone mass was assessed by $\mu \mathrm{CT}$. Scale bars, $50 \mu \mathrm{m}$. b Serum levels of the c-terminal telopeptide (CTX)- 1 in sham and OVX mice. c Immunohistochemistry analysis of USP53 (phycoerythrin [PE]; red fluorescence) and OCN (fluorescein isothiocyanate [FITC]; green fluorescence) with DAPI counterstaining in sham-operated and OVX mice (left). Quantification of IHC analysis (right). Scale bars, $20 \mu \mathrm{m}$. Results are means \pm SDs. ${ }^{*} P<0.01$ by an unpaired two-tailed Student's $t$-test. $n=5-10$ per group. $\mathbf{d}$ Diagram showing the molecular mechanism through which FBXO31 and USP53 regulate the Wnt signaling mediator, $\beta$-catenin, during the osteogenic differentiation of hBMSCs.

it is overexpressed around $50 \%$ in human prostate cancers and affects the invasion ability of prostate cells ${ }^{31}$. USP22 is also upregulated in many cancer cells and activates the proliferation, migration, and invasion of gastric cancer, colorectal cancer, and breast cancer ${ }^{32-34}$. Another study reported that USP4 is overexpressed in hepatocellular carcinoma (HCC) tissues. And, USP4 increases progression of HCC through stabilization of cyclophilin A and deubiquitination ${ }^{35}$. Recently, USP34 has been reported as a positive regulator in osteogenic differentiation of hBMSCs $^{36}$. Additionally, in the study about USP53, it was overexpressed in cervical cancer tissues and the inhibition of USP53 reduced cancer cell arrest ${ }^{37}$. But, none of the studies has reported about deubiquitinase enzyme which is related to osteoporosis. Here, we found that USP53 positively regulated the osteogenesis of hBMSCs in vitro and in vivo. Additionally, we confirmed that USP53 overexpression promoted osteogenesis in hBMSCs by activating the $\mathrm{Wnt} / \beta$-catenin signaling pathway in vitro and that USP53 bound with FBXO31 during the osteogenic differentiation of hBMSCs. Mechanistically, FBXO31 negatively regulated the osteogenic differentiation of hBMSCs by 
acting as an E3 ubiquitin ligase and degrading $\beta$-catenin. Finally, USP53 expression was decreased in patients with osteoporosis and in a mouse model of estrogen deficiencyinduced osteoporosis. Our findings provided further insights into the roles of DUBs in the pathogenesis of skeletal diseases and will be valuable for the development of novel therapeutic strategies for bone regeneration in tissue engineering.

We identified FBXO31 as a binding partner of USP53 and described its function in the osteogenesis of hBMSCs. F-box proteins (FBPs) are core components of E3 ubiquitin ligases and determine substrate specificity for degradation by protein targets ${ }^{38}$. Because the F-box motif is essential for the interactions within the SCF complex, this motif mediates the degradation of target proteins ${ }^{39}$. FBXO31 is a member of the FBP family and interacts with the SCF complex to form a functional SCF-FBXO31 E3 ubiquitin ligase, which regulates the ubiquitination of target proteins, such as mouse double minute 2, cyclin D1, forkhead box 1, chromatin licensing and DNA replication factor 1, Slug, mitogen-activated protein kinase kinase 6 , and Par6 $\mathrm{c}^{26,40}$. FBXO31 has been identified as a putative tumor-suppressor gene in breast, ovarian, hepatocellular, and prostate cancers. Its inactivation, due to heterozygosity loss, is associated with several cancers ${ }^{41}$. Additionally, FBXO31 has been reported to interact with the Skp1 protein ${ }^{26}$, and Cullin-1 and Roc-1 interact with mycFBXO31/HA-Skp1 ${ }^{26}$. Therefore, we hypothesized that FBXO31 may regulate the osteogenic differentiation of hBMSCs by modulating osteogenesis-related genes. Because the overexpression of USP53 by lentivirus results in enhanced osteogenic differentiation of hBMSCs through the $\mathrm{Wnt} / \beta$-catenin signal pathway, $\beta$-catenin may be a target of the SCF-FBXO31 E3 ubiquitin ligase $^{42,43}$. Here, we confirmed that SCF-FBXO31 bound with $\beta$-catenin and degraded $\beta$-catenin in hBMSCs. Moreover, FBXO31 mutant did not promoted the proteasomal degradation of $\beta$-catenin. Thus, FBXO31-SCF complex interaction was removed in FBXO31 mutant. Moreover, siFBXO31 enhanced the expression of active $\beta$-catenin and promoted the osteogenesis of hBMSCs. Conversely, overexpression of FBXO31 decreased active $\beta$-catenin and RUNX2 levels, thereby suppressing hBMSC osteogenesis. These results suggested that FBXO31 acted as an E3 ubiquitin ligase to degrade $\beta$-catenin, which then negatively regulated hBMSC osteogenesis. Accordingly, the FBXO31/ $\beta$-catenin/USP53 axis may positively regulate the osteogenic differentiation of hBMSCs (Fig. 7d).

To date, AAV is considered the most promising vector for gene therapy. AAV is a nonenveloped parvovirus with a single-stranded DNA genome $4.7 \mathrm{~kb}$ in length. This genome encodes four nonstrucutural replication proteins (Rep78, Rep68, Rep52, and Rep40) and three structural capsid proteins (viral proteins $1-3)^{44-46}$. The most commonly used AAV serotype in bone biology is AAV2, which is a potential carrier for target gene delivery in the bone tissue ${ }^{47-50}$. Recently, AAV9 was classified as an attractive vector for therapeutic gene delivery in bone tissue $e^{4,51,52}$. In this study, we investigated the effects of USP53 overexpressed in hBMSCs by delivering AAV2 in a mouse calvarial defect model. These mice showed better regeneration, as characterized by increased BV/TV, bone surface density, and mineral apposition rates in comparison with control mice. However, marked bone regeneration was not observed in AAV2-USP53-infected hBMSCs. These results may be related to the complete removal of the periosteum, which has bone regeneration capacity.

We also analyzed the USP53 expression in hBMSCs of elderly patients (79-94 years old) with osteoporosis using a GEO dataset (GSE35959) ${ }^{30}$. Notably, USP53 expression in patients with osteoporosis was downregulated compared with that in normal controls. Similar results were observed in OVX mice. Thus, these findings indicated that USP53 may play important roles in the pathogenesis of bone disorders such as osteoporosis.

In summary, USP53 acted as a positive regulator of hBMSC osteogenic differentiation through Wnt/ $\beta$-catenin signaling. The FBP, FBXO31, a component of the SCF complex, was shown to modulate the osteogenic differentiation of hBMSCs by acting as an E3 ubiquitin ligase to mediate the degradation of $\beta$-catenin. Intriguingly, USP53 was also shown to bind with the SCF ubiquitin ligase complex to reduce the polyubiquitination of $\beta$-catenin with FBXO31 dependently. Notably, transplantation of AAV2-USP53-infected hBMSCs improved bone regeneration in a mouse calvarial defect model. Taken together, our findings provide insights into the development of novel therapeutic strategies for the treatment of bone diseases. However, our findings are limited because we did not examine whether USP53 mutation/deletion was present in patients with osteoporosis. Further investigation is needed to determine the roles of USP53 in osteoblast lineages using Prx1-Cre;Usp53 $3^{\mathrm{f} / \mathrm{f}}, \operatorname{Sp} 7-\mathrm{Cre} ; U s p 53^{\mathrm{f} / \mathrm{f}}$, or Ocn-Cre;Usp $53^{\mathrm{f} / \mathrm{f}}$ mice.

\section{Materials and methods \\ Cell culture and reagents}

This study was approved by the Institutional Review Board of Yonsei University College of Medicine (IRB no. 4-2017-0232). Human bone marrow aspirates were obtained from the point $3 \mathrm{~cm}$ posterior to the anterior superior iliac spine of 10 healthy adult donors with a mean age of 35 years (range: 30-40 years). hBMSCs were maintained in low-glucose Dulbecco's modified Eagle's medium (DMEM-LG; Gibco BRL, Rockville, MD, USA) with $10 \%$ fetal bovine serum (FBS; Gibco) and $1 \%$ antibiotic-antimycotic solution (Gibco). Human embryonic kidney 293 (HEK293) and 293FT cells were obtained 
from American Type Culture Collection (Manassas, VA, USA). These cells were maintained in high-glucose DMEM (DMEM-HG; Gibco) containing 10\% FBS and 1\% antibiotic-antimycotic solution (Gibco). DKK1 (R\&D Systems) was used at a concentration of $0.1 \mathrm{mg} / \mathrm{mL}$ in hBMSC $^{53}$. Wnt3a (cat. no. 5036-WN; R\&D Systems) was used at a concentration of $10 \mathrm{ng} / \mathrm{mL}$ in $\mathrm{hBMSCs}^{54,55}$.

\section{Microarray re-analysis}

The mRNA expression profiles of hBMSCs in osteogenic differentiation medium were found in GEO datasets (https://www.ncbi.nlm.nih.gov/geo/) based on GSE18043 ${ }^{24}$. A web-based tool, GEO2R (http://www.ncbi.nlm.nih.gov/ geo/geo2r/) was used to analyze the DEGs by comparing up-regulated DUBs during hBMSC osteogenesis at different time points (day 0 versus days 1,3 , or 7 ). The cut-off value was set as $P<0.05, \log$ (fold change) $>2.0$. The mRNA expression profiles of hBMSCs from five patients with osteoporosis (79-94 years old) were found in GEO datasets based on GSE35959 ${ }^{30}$ that contained a total of 9 samples (hMSCs derived from 3 normal females, 1 normal male donor, and 5 osteoporosis female patients). The cut-off value was set as $P<0.05$, log (fold change) $<0.5$.

\section{Osteogenic differentiation of hBMSCs}

hBMSCs were seeded onto 12-well culture plates at a density of $8 \times 10^{4}$ cells/well and then maintained for 10 days in osteogenic medium (DMEM-LG containing $10 \%$ FBS, $1 \%$ antibiotic-antimycotic solution, $100 \mathrm{nM}$ dexamethasone, $10 \mathrm{mM} \beta$-glycerophosphate, and $50 \mu \mathrm{g} / \mathrm{mL}$ ascorbic acid $)^{53}$. The osteogenic medium was replenished every 3 days. Alizarin red S and alkaline phosphatase (ALP) staining procedures were performed as previously described $^{44}$.

\section{Quantitative real-time reverse transcription polymerase chain reaction (qRT-PCR)}

Total RNA was isolated from cells using an RNeasy kit (cat. no. 74106; Qiagen, Valencia, CA, USA). RNA ( $2 \mu \mathrm{g}$ ) was then reverse transcribed using an Omniscript kit (cat. no. 205113; Qiagen). qRT-PCR was performed using a StepOnePlus Real-Time PCR System (Applied Biosystems, Foster City, CA, USA) and $2 \times$ qPCRBIO SyGreen Mix (cat. no. PB20.12-05; PCR Biosystems) according to the manufacturers' guidelines. Primer sequences are listed in Supplementary Table S5.

\section{Western blotting}

Total proteins were prepared from cells using passive lysis buffer (cat. no. E194A; Promega, Madison, WI, USA), and $30 \mu \mathrm{g}$ of each sample was separated by sodium dodecyl sulfate polyacrylamide gel electrophoresis (SDSPAGE) and transferred to polyvinylidene difluoride membranes. After blocking with tris-buffered saline with
Tween 20 containing 5\% skim milk, blots were incubated overnight at $4{ }^{\circ} \mathrm{C}$ with primary antibodies. All membranes were then incubated with horseradish peroxidaseconjugated secondary antibodies (1:5000; GenDEPOT) for $1 \mathrm{~h}$ at room temperature. Finally, the western blots were imaged using LAS 4000 (Fujifilm). Primary antibodies are listed in Supplementary Table S6.

\section{Plasmids and RNA interference}

FLAG-HA-USP53 was gifted by Wade Harper (Addgene plasmid \#22606). FLAG- $\beta$-catenin was a gift from Eric Fearon (Addgene plasmid \#16828). pCMV-Myc (Clontech plasmid \#631604) was purchased from Clontech (TaKaRa, Shiga, Japan). myc-F-box only protein 31 (FBXO31) and myc-FBXO31 $\triangle \mathrm{F}$ were kind gifts from Prof. David F. Callen (University of Adelaide and Hanson Institute, Adelaide, Australia). The FBOX31 clone (untagged) was provided by the Korea Human Gene Bank, Medical Genomics Research Center (KRIBB, Daejeon, South Korea). Green fluorescent protein (GFP)-RUNX2, myc-Ub, HA-Ub, and HA-Ub(K48) were kindly provided by Prof. Jae-Hyuck Shim (University of Massachusetts Medical School, Worcester, MA, USA). Lentiviral control vector (pLenti-GIII-CMV-GFP-2A-Puro, LV590) and lentiviral vector-USP53 (pLenti-GIII-CMVUSP53-GFP-2A-Puro, LV353779) were purchased from Applied Biological Materials (Richmond, BC, Canada). Scramble negative control small interfering RNA (siRNA; siRNA no. SN1002), USP53 siRNA (siRNA no. 54532), and FBXO31 siRNA (siRNA no. 79791) were purchased from Bioneer (Daejeon, South Korea).

\section{Lentivirus production}

293FT cells were transfected with USP53-overexpressing lentivirus or USP53 shRNA along with packaging plasmid (delta 8.9) and envelop plasmid (VSV-G) using Lipofectamine LTX \& Plus Reagent (Invitrogen, Carlsbad, CA, USA) according to the manufacturer's instructions. DMEM-LG supplemented with $10 \%$ FBS was changed $6 \mathrm{~h}$ after transfection. The supernatants containing virus were collected 24 and $48 \mathrm{~h}$ after initial transfection, filtered with a $0.45-\mu \mathrm{m}$ syringe filter (Millipore), separated into aliquots, and stored at $-80^{\circ} \mathrm{C}$ until use. hBMSCs were seeded onto 6-well plates at a density of $1 \times 10^{5}$ cells/well and transfected with purified lentiviruses for $24 \mathrm{~h}$. Puromycin $(10 \mu \mathrm{g} / \mathrm{mL}$; Sigma, St. Louis, MO, USA) was used for selection, and stable lentivirus-infected hBMSCs were used in experiments. Information on the lentiviral vectors is provided in Supplementary Table S7.

\section{Adeno-associated virus transduction}

AAV blank control with GFP (serotype 2, $10^{9}$ genome copies $[\mathrm{GC}] / \mathrm{mL}$, AAV2-control, AAVP022) and AAVUSP53 with GFP (serotype 2, $10^{9} \mathrm{GC} / \mathrm{mL}$, AAV2-USP53, AAVP4915843) were purchased from Applied Biological 
Materials. For AAV infection, hBMSCs were seeded onto 12 -well plates at a density of $8 \times 10^{4}$ cells/well. After $24 \mathrm{~h}$, hBMSCs were incubated with $40 \mu \mathrm{L}$ AAV2-control and AAV2-USP53 using X-tremeGENE ${ }^{\mathrm{TM}_{9}}$ DNA transfection reagent (Sigma). AAVs infected with hBMSCs were grown to $80 \%$ confluence and then replated at a density of $1 \times 10^{5}$ cells in a $10-\mathrm{cm}^{2}$ dish.

\section{Immunoprecipitation analysis}

Transfected cells were incubated with $10 \mu \mathrm{M}$ MG132 (cat. no. M7749; Sigma) for $6 \mathrm{~h}$, and whole cells were lysed using nondenaturing lysis buffer $(20 \mathrm{mM}$ Tris- $\mathrm{HCl}[\mathrm{pH}$ 8.0], $137 \mathrm{mM} \mathrm{NaCl}, 0.5 \%$ Nonidet $\mathrm{P} 40$ with $2 \mathrm{mM}$ ethylenediaminetetraacetic acid [EDTA, $\mathrm{pH}$ 8.0], $1 \mathrm{mM}$ phenylmethylsulfonyl fluoride, and protease inhibitor). The lysates were incubated with anti-myc or HA antibodies overnight at $4{ }^{\circ} \mathrm{C}$. Then, lysates were conjugated with $40 \mu \mathrm{L}$ pre-washed protein A/G agarose beads (Santa Cruz Biotechnology, Santa Cruz, CA, USA) for $4 \mathrm{~h}$ at $4{ }^{\circ} \mathrm{C}$ with gentle rotation. Immunoprecipitates were eluted with $2 \times$ lane marker-reducing sample buffer (Thermo Fisher Scientific). Eluates were separated using SDS-PAGE.

\section{Liquid chromatography tandem mass spectrometry (LC-MS/MS)}

To investigate USP53-binding proteins in hBMSCs, the cells were transfected with lentiviral control vector or lentiviral vector-USP53 and cultured in osteogenic induction medium for 4 days. The cells were then treated with MG132 $(10 \mu \mathrm{M})$ for $6 \mathrm{~h}$, and hBMSCs were harvested using passive lysis buffer (Promega). Total protein (approximately $500 \mu \mathrm{g}$ ) was incubated with an antibody against GFP (cat. no. sc-390394; Santa Cruz Biotechnology) and IgG control (cat. no. 12-370; Milipore) at $4{ }^{\circ} \mathrm{C}$ overnight. SDS-PAGE was performed, following which the gels were stained with an InstantBlue staining kit (cat. no. ISB1L; Sigma). Differential bands were collected for LCMS/MS, which was performed using a LTQ orbitrap mass spectrometer (Thermo Electron, San Jose, CA, USA).

\section{Luciferase reporter assays}

hBMSCs were seeded onto 6-well plates at a density of $1 \times 10^{5}$ cells/well, and cells were transfected with $1 \mu \mathrm{g}$ pCMV-myc, myc-FBXO31, myc-FBXO31 $\Delta \mathrm{F}$ plus TOPflash (wild-type $\mathrm{T}$ cell factor binding site), or FOP-flash (mutant T cell factor binding site) using Lipofectamine LTX \& Plus Reagent (Invitrogen) according to the manufacturer's instructions. After $24 \mathrm{~h}$, the cells were maintained for 5 days in osteogenic induction medium. The cells were analyzed using a simple dual-luciferase assay (Promega).

\section{Protein degradation assay}

hBMSCs were seeded at a density of $1 \times 10^{5}$ cells/well onto 6-well plates and transfected with Flag- $\beta$-catenin and control or Flag-USP53. Cells were cultured in the presence of Wnt3a. After $48 \mathrm{~h}, 100 \mu \mathrm{g} / \mathrm{mL}$ cycloheximide (cat. no. C4859; Sigma) was added. Cells were harvested at the indicated time points.

\section{Animal experiments}

The Committee on the Ethics of Animal Experiments of Yonsei University College of Medicine approved all animal experiments and protocols (permit no. IACUC-20190168). Fifteen male Institute of Cancer Research (ICR) mice ( 8 weeks old) were anesthetized with zoletil (30 $\mathrm{mg} / \mathrm{kg}$ body weight; Virbac Laboratories) and rompun (10 mg/kg body weight; Bayer Korea) by intraperitoneal injection. After shaving the skull, an alcohol swab was used before hair trimming. The skin was retracted. Critical-sized calvarial bone defects $(4 \mathrm{~mm}$ inside diameter $/ 5 \mathrm{~mm}$ outside diameter) were created using a trephine burr (Shudent). The animals were divided into three groups as follows: (1) fibrin gel only, (2) hBMSCs $\left(5 \times 10^{5}\right)$ with AAV2-control group, and (3) hBMSCs $\left(5 \times 10^{5}\right)$ with AAV2-USP53. Fibrin gel $(20 \mu \mathrm{L}$; TISSEEL $)$ was mixed with hBMSCs and implanted into defects. Finally, the defect site was closed with vicryl plus (Ethicon 3-0) and nylon (Dermalon 3-0). Metacam (1 mg/kg) was used as an analgesic. Mice were sacrificed 8 weeks after surgery, and the skulls were harvested.

\section{Micro-computed tomography $(\mu \mathrm{CT})$ and histological analysis}

Murine skulls were fixed in $4 \%$ paraformaldehyde (PFA) for 3 days, following which $\mu \mathrm{CT}$ was performed using a Skyscan 1173 High Energy Micro-CT scanner (Skyscan NV, Kontich, Belgium) with a $40-130 \mathrm{kV}, 8 \mathrm{~W}$ $\mathrm{X}$-ray source. For histological analysis, skulls or femurs were decalcified with $5 \%$ formic acid (Sigma) or $0.5 \mathrm{M}$ EDTA for 1 week. Fixed specimens were then embedded in paraffin blocks and cut into $4-\mu \mathrm{m}$-thick sections. Sections were deparaffinized in xylene and serially rehydrated in ethanol. Sections were sequentially stained with Masson trichrome (M\&T) staining and immunohistochemistry (IHC). Primary antibodies are listed in Supplementary Table S8. For, 3,3'-diaminobenzidine tetrahydrochloride staining, we used a Dako REAL EnVision Detection System (cat. no. K5007; DAKO), and counterstaining was performed with hematoxylin (cat. no. GHS-3; Sigma). A Zeiss LSM 700 confocal laser scanning microscope (Carl Zeiss Micro Imaging $\mathrm{GmbH}$, Jena, Germany) was used to visualize images. For performing mouse calvarial bone histomorphometry, $25 \mathrm{mg} / \mathrm{kg}$ calcein (cat. no. C0875; Sigma) dissolved in $2 \%$ sodium bicarbonate solution was subcutaneously injected into mice at 4-day intervals (days 6 and 2 before sacrifice). Undecalcified skull samples were fixed in 4\% PFA for 3 days and then embedded in plastic 
resin blocks (methyl methacrylate) for obtaining bone sections $(7 \mu \mathrm{m})$.

\section{Enzyme-linked immunosorbent assay (ELISA)}

The Committee on the Ethics of Animal Experiments of Yonsei University College of Medicine approved all animal experiments and protocols (permit no. IACUC-20200045). Female sham-operated and ovariectomized (OVX) mice (C57BL/6), 6 weeks old) were purchased from Japan SLC (Hamamatsu, Japan). After 8 weeks, the sham and OVX mice were sacrificed, and serum was collected by cardiac puncture. Mouse RatLaps (C-terminal telopeptide type I collagen [CTX-1]; cat. no. AC-06F1; IDS) was used according to the manufacturer's recommendations to measure serum level of CTX-1.

\section{Statistical analysis}

For each experiment, samples were analyzed at least in triplicate. Results with $P$ values less than 0.05 were considered statistically significant. All data are presented as means \pm standard deviations (SDs). No animals or samples were excluded from the analysis. We first performed Shapiro-Wilk normality tests for normal distributions of the groups. If normality tests were passed, two-tailed, unpaired Student's t-tests were used for the comparisons between two groups. For comparisons of three or four groups, we used one-way analysis of variance (ANOVA) if normality tests were passed, followed by Tukey's multiple comparison tests for all pairs of groups. GraphPad PRISM software (version 8.0; La Jolla, CA, USA) was used for statistical analysis.

\section{Acknowledgements}

We thank Prof. David F. Callen (University of Adelaide and Hanson Institute, Australia) for providing pCMV-myc-FBXO31 and F-box domain deletion mutation vector pCMV-myc-FBXO31 $\triangle F$. The authors thank Medical Illustration \& Design, part of the Medical Research Support Services of Yonsei University College of Medicine, for assistance in the preparation of artwork.

\section{Author details}

'Department of Orthopaedic Surgery, Yonsei University College of Medicine, 50-1 Yonsei-ro, Seodaemun-gu, Seoul 03722, South Korea. ${ }^{2}$ Brain Korea 21 PLUS Project for Medical Sciences, Yonsei University College of Medicine, 50-1 Yonsei-ro, Seodaemun-gu, Seoul 03722, South Korea. ${ }^{3}$ Severance Biomedical Science Institute, Yonsei University College of Medicine, 50-1 Yonsei-ro, Seodaemun-gu, Seoul 03722, South Korea

\section{Author contributions}

D.B. and K.H.P. conceived and designed the study and wrote the manuscript. K.-M.L. and K.H.P. performed the in vivo experiments and analyzed the data. S.J., S.J., and J.K. performed the development of revision of the revised paper. J.W.L. conceived and oversaw the project and wrote the manuscript.

\section{Ethics statement}

Human bone marrow-derived MSCs (hBMSCs) were obtained from the posterior iliac crest of ten adult donors (age range: 30 - 40 years) after receiving approval from the Institutional Review Board (IRB no. 4-2017-0232). All experiments with mice were performed in an Animal Care Center and in accordance with Association for Assessment and Accreditation of Laboratory Animal Care (AAALAC) International Guidelines. All animal care and use protocols were approved by the Committee on the Ethics of Animal Experiments.

\section{Funding statement}

This research was supported by Mid-Career Research Program through the National Research Foundation of Korea (NRF) funded by the Ministry of Education (NRF-2019R1A2C1087777) and from the Bio \& Medical Technology Development Program of the National Research Foundation funded by the Ministry of Science, ICT \& Future Planning (NRF-2017M3A9E8029722).

\section{Conflict of interest}

The authors declare no competing interests.

\section{Publisher's note}

Springer Nature remains neutral with regard to jurisdictional claims in published maps and institutional affiliations.

Supplementary information The online version contains supplementary material available at https://doi.org/10.1038/s41419-021-03517-x.

Received: 27 August 2020 Revised: 29 January 2021 Accepted: 4 February 2021

Published online: 04 March 2021

\section{References}

1. Bernard, N. J. Sensing bone mass. Nat. Rev. Rheumatol. 15, 128 (2019).

2. Ray, K. Bone: The immune system takes control of bone homeostasis. Nat. Rev. Rheumatol. 10, 382 (2014).

3. Modinger, Y., Loffler, B., Huber-Lang, M. \& Ignatius, A. Complement involvement in bone homeostasis and bone disorders. Semin Immunol. 37(C), 53-65 (2018).

4. Harada, S. \& Rodan, G. A. Control of osteoblast function and regulation of bone mass. Nature 423, 349-355 (2003).

5. Eastell, R. et al. Postmenopausal osteoporosis. Nat. Rev. Dis. Primers. 2, 16069 (2016).

6. Morris, J. A. et al. An atlas of genetic influences on osteoporosis in humans and mice. Nat. Genet. 51, 258-266 (2019).

7. Hopkins, R. B. et al. The relative efficacy of nine osteoporosis medications for reducing the rate of fractures in post-menopausal women. BMC Musculoskelet. Disord. 12, 209 (2011).

8. Khosla, S. \& Hofbauer, L. C. Osteoporosis treatment: recent developments and ongoing challenges. Lancet Diabetes Endocrinol. 5, 898-907 (2017).

9. Coleman, R. E. Risks and benefits of bisphosphonates. Br. J. Cancer 98, 1736-1740 (2008).

10. Borumandi, F., Aghaloo, T., Cascarini, L., Gaggl, A. \& Fasanmade, K. Antiresorptive drugs and their impact on maxillofacial bone among cancer patients. Anti-Cancer Agent Me 15, 736-743 (2015).

11. Lecker, S. H., Goldberg, A. L. \& Mitch, W. E. Protein degradation by the ubiquitin-proteasome pathway in normal and disease states. J. Am. Soc Nephrol. 17, 1807-1819 (2006).

12. Mevissen, T. E.T. \& Komander, D. Mechanisms of deubiquitinase specificity and regulation. Annu. Rev. Biochem. 86, 159-192 (2017).

13. Harrigan, J. A., Jacq, X., Martin, N. M. \& Jackson, S. P. Deubiquitylating enzymes and drug discovery: emerging opportunities. Nat. Rev. Drug Disco. 17, 57-78 (2018).

14. Severe, N., Dieudonne, F. X. \& Marie, P. J. E3 ubiquitin ligase-mediated regulation of bone formation and tumorigenesis. Cell Death Dis. 4, e463 (2013).

15. Kaneki, H. et al. Tumor necrosis factor promotes Runx2 degradation through up-regulation of Smurf1 and Smurf2 in osteoblasts. J. Biol. Chem. 281, 4326-4333 (2006).

16. Shu, L., Zhang, H., Boyce, B. F. \& Xing, L. Ubiquitin E3 ligase Wwp1 negatively regulates osteoblast function by inhibiting osteoblast differentiation and migration. J. Bone Min. Res. 28, 1925-1935 (2013).

17. Liu, J. et al. Ubiquitin E3 ligase Itch negatively regulates osteoblast function by promoting proteasome degradation of osteogenic proteins. Bone Jt. Res $\mathbf{6}$, 154-161 (2017).

18. Thacker, G. et al. Skp2 inhibits osteogenesis by promoting ubiquitinproteasome degradation of Runx2. Biochim Biophys. Acta 1863, 510-519 (2016).

19. Shim, J. H. et al. Schnurri-3 regulates ERK downstream of WNT signaling in osteoblasts. J. Clin. Invest. 123, 4010-4022 (2013). 
20. Tang, Y. M. et al. Protein deubiquitinase USP7 is required for osteogenic differentiation of human adipose-derived stem cells. Stem Cell Res. 8, 186 (2017).

21. Guo, Y. C. et al. Ubiquitin-specific protease USP34 controls osteogenic differentiation and bone formation by regulating BMP2 signaling. Embo J. 37, e99398 (2018)

22. Kazmierczak, M. et al. Progressive hearing loss in mice carrying a mutation in Usp53. J. Neurosci. 35, 15582-15598 (2015).

23. Maddirevula, S. et al. Identification of novel loci for pediatric cholestatic liver disease defined by KIF12, PPM1F, USP53, LSR, and WDR83OS pathogenic variants. Genet. Med. 21, 1164-1172 (2019).

24. Hamidouche, Z. et al. Priming integrin alpha5 promotes human mesenchymal stromal cell osteoblast differentiation and osteogenesis. Proc. Natl Acad. Sci. USA 106, 18587-18591 (2009).

25. Johansson, P. et al. SCF-FBXO31 E3 ligase targets DNA replication factor Cdt1 for proteolysis in the $\mathrm{G} 2$ phase of cell cycle to prevent re-replication. J. Biol. Chem. 289, 18514-18525 (2014).

26. Kumar, R. et al. FBXO31 is the chromosome 16q24.3 senescence gene, a candidate breast tumor suppressor, and a component of an SCF complex. Cancer Res. 65, 11304-11313 (2005)

27. Kramer, I. et al. Osteocyte Wnt/beta-catenin signaling is required for normal bone homeostasis. Mol. Cell Biol. 30, 3071-3085 (2010).

28. Gaur, T. et al. Canonical WNT signaling promotes osteogenesis by directly stimulating Runx2 gene expression. J. Biol. Chem. 280, 33132-33140 (2005).

29. Paul, D. et al. F-box protein FBXO16 functions as a tumor suppressor by attenuating nuclear beta-catenin function. J. Pathol. 248, 266-279 (2019).

30. Benisch, P. et al. The transcriptional profile of mesenchymal stem cell populations in primary osteoporosis is distinct and shows overexpression of osteogenic inhibitors. PLOS ONE. 7, e45142 (2012).

31. Benassi, B. et al. MYC is activated by USP2a-mediated modulation of microRNAs in prostate cancer. Cancer Discov. 2, 236-247 (2012).

32. Ma, Y. et al. USP22 maintains gastric cancer stem cell stemness and promotes gastric cancer progression by stabilizing BMl1 protein. Oncotarget 8, 33329-33342 (2017).

33. Kim, D. et al. Deubiquitinating enzyme USP22 positively regulates c-Myc stability and tumorigenic activity in mammalian and breast cancer cells. J. Cell. Physiol. 232, 3664-3676 (2017)

34. Ji, M. et al. Ubiquitin specific protease 22 promotes cell proliferation and tumor growth of epithelial ovarian cancer through synergy with transforming growth factor $\beta 1$. Oncol. Rep. 33, 133-140 (2015).

35. Li, T. et al. Ubiquitin-specific protease 4 promotes hepatocellular carcinoma progression via cyclophilin A stabilization and deubiquitination. Cell death Dis. 9, 148 (2018).

36. Pathria, G. et al. Targeting the Warburg effect via LDHA inhibition engages ATF4 signaling for cancer cell survival. EMBO J. 37, e99735 (2018).

37. Zhou, Q., Yao, X., Wu, C., Chen, S. \& Fan, D. Knockdown of ubiquitin-specific protease 53 enhances the radiosensitivity of human cervical squamous cell carcinoma by regulating DNA damage-binding protein 2. Technol. Cancer Res. Treat. 19, 1533033820929792 (2019)
38. Gong, J. \& Huo, J. R. New insights into the mechanism of F-box proteins in colorectal cancer (Review). Oncol. Rep. 33, 2113-2120 (2015).

39. Malonia, S. K., Dutta, P., Santra, M. K. \& Green, M. R. F-box protein FBXO31 directs degradation of MDM2 to facilitate p53-mediated growth arrest following genotoxic stress. P Natl Acad. Sci. USA 112, 8632-8637 (2015).

40. Tan, Y., Liu, D., Gong, J., Liu, J. \& Huo, J. The role of F-box only protein 31 in cancer. Oncol. Lett. 15, 4047-4052 (2018).

41. Dutta, P. et al. The tumor suppressor FBXO31 preserves genomic integrity by regulating DNA replication and segregation through precise control of cyclin A levels. J. Biol. Chem. 294, 14879-14895 (2019).

42. Kimelman, D. \& Xu, W. beta-catenin destruction complex: insights and questions from a structural perspective. Oncogene 25, 7482-7491 (2006).

43. Su, Y., Ishikawa, S., Kojima, M. \& Liu, B. Eradication of pathogenic beta-catenin by Skp1/Cullin/F box ubiquitination machinery. Proc. Natl Acad. Sci. USA 100, 12729-12734 (2003)

44. Yang, Y. S. et al. Bone-targeting AAV-mediated silencing of Schnurri-3 prevents bone loss in osteoporosis. Nat Commun. 10, 2958 (2019).

45. Gao, G., Vandenberghe, L. H. \& Wilson, J. M. New recombinant serotypes of AAV vectors. Curr. Gene Ther. 5, 285-297 (2005).

46. Becerra, S. P., Koczot, F., Fabisch, P. \& Rose, J. A. Synthesis of adeno-associated virus structural proteins requires both alternative messenger-rna splicing and alternative initiations from a single transcript. J. Virol. 62, 2745-2754 (1988).

47. Chen, $Y$. et al. Gene therapy for new bone formation using adeno-associated viral bone morphogenetic protein-2 vectors. Gene Ther. 10, 1345-1353 (2003).

48. Kang, $Y$. et al. In vitro and in vivo induction of bone formation based on adeno-associated virus-mediated BMP-7 gene therapy using human adiposederived mesenchymal stem cells (vol 28, pg 839, 2007). Acta Pharm. Sin. 28, 1044- (2007).

49. Gafni, Y. et al. Gene therapy platform for bone regeneration using an exogenously regulated, AAV-2-based gene expression system. Mol. Ther. 9, 587-595 (2004)

50. Choi, V. W., McCarty, D. M. \& Samulski, R. J. AAV hybrid serotypes: improved vectors for gene delivery. Curr. Gene Ther. 5, 299-310 (2005).

51. Lee, L. R. et al. Targeting Adeno-Associated Virus Vectors for Local Delivery to Fractures and Systemic Delivery to the Skeleton. Mol. Ther. Methods Clin. Dev. 15, 101-111 (2019).

52. Luo, F. T. et al. Adeno-Associated Virus-Mediated RNAi against Mutant Alleles Attenuates Abnormal Calvarial Phenotypes in an Apert Syndrome Mouse Model. Mol. Ther.-Nucl. Acids 13, 291-302 (2018).

53. Park, K. H. et al. Zinc Promotes Osteoblast Differentiation in Human Mesenchymal Stem Cells Via Activation of the CAMP-PKA-CREB Signaling Pathway. Stem cells Dev. 27, 1125-1135 (2018).

54. Lai, W. T., Krishnappa, V. \& Phinney, D. G. Fibroblast growth factor 2 (Fgf2) inhibits differentiation of mesenchymal stem cells by inducing Twist2 and Spry4, blocking extracellular regulated kinase activation, and altering Fgf receptor expression levels. Stem cells (Dayt., Ohio) 29, 1102-1111 (2011).

55. Kim, J. A., Choi, H. K., Kim, T. M., Leem, S. H. \& Oh, I. H. Regulation of mesenchymal stromal cells through fine tuning of canonical Wnt signaling. Stem cell Res. 14, 356-368 (2015). 